\title{
Paraganglioma não-funcionante de bexiga: relato de caso e revisão da literatura
}

\author{
Non-functioning paraganglioma of the bladder: case report and literature review
}

Ulysses Messias da Silva', Ricardo Alexandre Manteuffel ${ }^{2}$, Mônica Serapião ${ }^{3}$, Roberto Carlos Sedrez ${ }^{4}$

\begin{abstract}
Resumo
Introdução: O s paragangliomas são feocromocitomas de localização extra-adrenal, podendo ser encontrados desde a base do crânio até a bexiga. Representam menos de 0,06\% de todos os tumores da bexiga, sendo os primeiros casos publicados por Zimmermann em 1953, e por Rodriguez-Rubio em 1975. Relato do caso: Paciente de 17 anos, masculino, deu entrada no Hospital Regional Alto Vale apresentando hematúria e retenção urinária. Foi investigado sem achados positivos, tendo recebido alta hospitalar para investigação ambulatorial do quadro. Diagnosticado 0 tumor vesical ambulatorialmente, o paciente foi internado para tratamento cirúrgico eletivo do tumor, cujo exame anátomo-patológico revelou a presença de um paraganglioma, confirmado após imunohistoquímica. O nze meses após a cirurgia o paciente permanece hígido e assintomático. D iscussão: A idade de apresentação oscila entre os 11 e os 70 anos. Clinicamente são classificados em funcionantes e não-funcionantes de acordo com a presença de produção catecolaminérgica pela neoplasia. D ez a quinze por cento dos paragangliomas de bexiga são malignos. 0 diagnóstico é confirmado pela dosagem do Ácido Vanilmandélico e M etanefrinas urinárias, U Itrassonografia, Urografia Excretora, Cistoscopia, Tomografia Computadorizada, Ressonância N uclear M agnética e a Cintilografia com lodo 131-M etaiodobenzilguanidina, sendo este último apontado como o mais sensível pela literatura. 0 tratamento é cirúrgico, e o prognóstico ainda é uma incógnita devido ao ainda pequeno número de casos relatados.

Palavras-chave: Paraganglioma; N eoplasias da bexiga; Feocromocitoma; Tumor vesical.
\end{abstract}

\footnotetext{
${ }^{1}$ M édico Residente do segundo ano do Programa de Residência M édica em Cirurgia Geral do Hospital Regional Alto Vale, Santa Catarina, Brasil. ${ }^{2}$ M édico Residente do segundo ano do Programa de Residência M édica em Cirurgia Geral do Hospital Regional Alto Vale, Santa Catarina, Brasil.

${ }^{3}$ M édica Patologista do Serviço de Anatomia-Patológica do H ospital Regional Alto Vale, Santa Catarina, Brasil.

${ }^{4}$ M édico U rologista do Serviço de U rologia do H ospital Regional Alto Vale, Santa Catarina, Brasil.

§ Trabalho científico desenvolvido durante o Programa de Residência M édica em Cirurgia Geral do H ospital Regional Alto Vale, Santa Catarina, Brasil.

Endereço para correspondência: R. A. M. Rua Dionísio Cerqueira, 400, Saguaçu; 89221-160. Joinville - SC, Brasil.
} 


\begin{abstract}
Introduction: Paragangliomas are pheochromocytomas with an extra-adrenal location. They can be found between the base of the skull and the bladder. They account for less than $0.06 \%$ of all bladder tumors, and were first reported by Zimmermann in 1953, and Rodriguez-Rubio in 1975. Case report: A 17 year old male patient was admitted to Alto Vale Regional Hospital with hematuria and urinary retention. He was examined but nothing positive was found, after which he was discharged for outpatient treatment. After the vesical tumor was diagnosed, the patient was admitted to $\mathrm{H}$ ospital for elective surgical treatment of the tumor. After this, pathological analysis revealed a paraganglioma, confirmed by immunohistochemistry. Eleven months after surgery, the patient remains healthy and assymptomatic. D iscussion: Paragangliomas usually appear between 11 and 70 years, and are clinically classified as functioning or non-functioning depending on cathecolaminergic production by the tumor. Ten to fifteen percent of bladder paragangliomas are malignant. The diagnosis is confirmed by urinary vanilmandelic acid and urinary metanephryns, ultrassonography, excretory urography, cistoscopy, computerized tomography, magnetic nuclear resonance, and 131-metaiodobenzilguanidine iodine cintilography, with the last one being the most sensitive according to the literature. The treatment is surgical, and the prognosis is uncertain due to small number of cases reported.
\end{abstract}

Key words Paraganglioma; Bladder neoplasms; Pheochromocytoma; Vesical tumor.

\section{INTRODUÇÃO}

Os feocromocitomas são tumores originários das células cromafins, que são células especializadas não epiteliais da crista neural, associadas aos gânglios simpáticos na vida fetal ${ }^{1}$. A pós o nascimento, a maioria destas células se degeneram e o maior número de células cromafins residuais permanece na medula adrenal ${ }^{1}$. Assim sendo, os paragangliomas são feocromocitomas de localização extra-adrenal 1,3,4. Estes tumores podem se desenvolver em qualquer lugar onde existirem células cromafins, onde sua maior concentração é na medula adrenal. Todavia, podem ser encontrados desde a base do crânio até a bexiga3. 0 paragânglio caracteriza-se pela presença de depósitos granulares de células principais que contêm substâncias neurotransmissoras, que provavelmente exercem a função comum de governar níveis locais de excitação dentro do sistema nervoso autônomo ${ }^{1}$.

O s paragangliomas de bexiga são tumores raros, que representam menos de $0,06 \%$ de todos os tumores da bexiga ${ }^{1}$, sendo os primeiros casos publicados por Zimmermann em 1953, e na Espanha por RodriguezRubio em 1975, e a partir de então são referidos na literatura cerca de $200 \operatorname{casos}^{2,3,4}$. Sua capacidade de invasão justifica sua classificação como tumor maligno, mas achados histopatológicos sugerem um curso relativamente benigno ${ }^{2}$. Ainda assim, alguns autores consideram a localização um critério prognóstico de maior valor do que o estudo convencional ${ }^{4}$.

\section{RELATO DE CASO}

J.S., 17 anos, masculino, branco, solteiro, estudante, natural de Petrolândia - Santa C atarina (SC), procedente de Atalanta - SC, deu entrada no Pronto Socorro do H ospital Regional Alto Vale no dia 09 de abril de 2001 com queixas de hematúria e retenção urinária. Apresentava-se há 14 horas com hematúria macroscópica e eliminação de coágulos, seguida de retenção urinária por tamponamento vesical. Referiu episódio de hematúria há 30 meses, tendo sido investigado sem achados positivos. Foi internado, permanecendo hospitalizado até o dia 13 e abril de 2001. $\mathrm{N}$ este período foi submetido à sondagem vesical com irrigação contínua, e feitos os seguintes estudos laboratoriais: hemograma, parcial de urina, urocultura, creatinina sérica, tempo de protrombina (TP), tempo de tromboplastina parcial ativada (KPTT), tempo de coagulação e tempo de sangramento. Todos sem alterações, exceto o parcial de urina por impossibilidade de análise pela hematúria intensa, o hemograma que apresentou 13.300 leucócitos/mm3 e 04\% debastonetes, com urocultura sem crescimento bacteriano. 0 paciente também foi submetido à ultrassonografia abdominal e à cistoscopia. A ultrassonografia evidenciou alteração apenas na bexiga, que apresentava paredes irregulares, contendo material heterogêneo em seu assoalho, sugerindo tratar-se de coágulos envolvendo o balão de Foley (figura 1). $\mathrm{N}$ a cistoscopia não foi possível visualizar as paredes da bexiga devido à presença de urina muito 


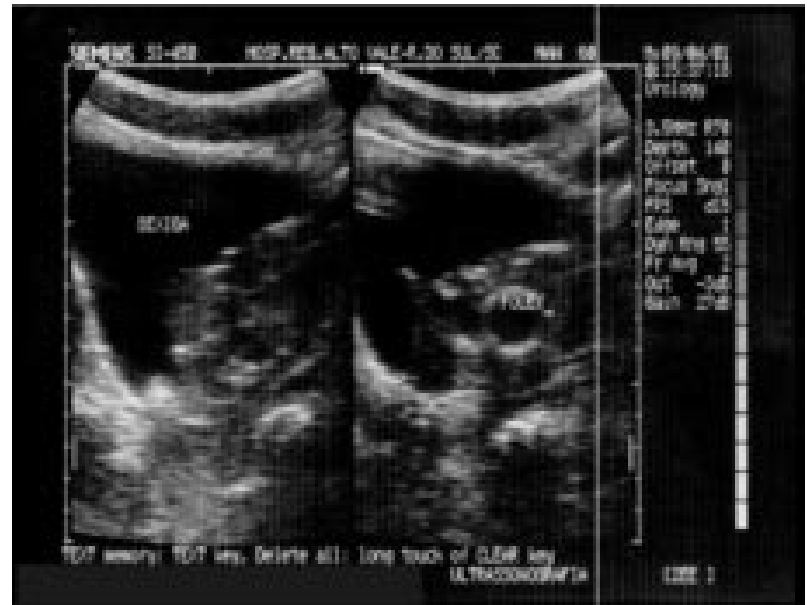

Figura 1 - Ultrassonografia mostrando bexiga com paredes irregulares e coágulos envolvendo o balão de Foley.

\section{turva e coágulos.}

N este período apresentou-se assintomático e com sinais vitais estáveis, tendo alta hospitalar no dia 13 de abril de 2001, urinando bem, sem dificuldades, com urina clara, e com orientação para investigação ambulatorial. Retornou dia 20 de abril de 2001 onde realizou cistoscopia ambulatorial (figura 2) tendo sido feito o diagnóstico de tumor de bexiga.

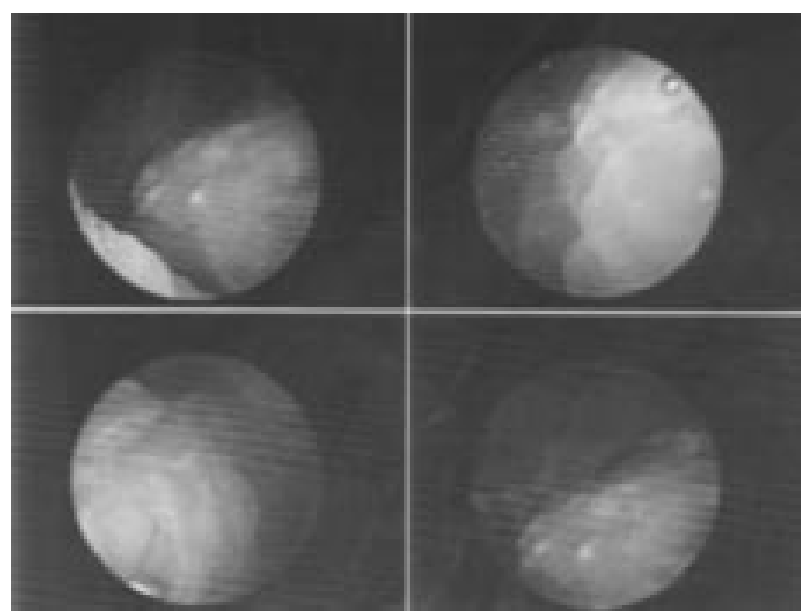

Figura 2 - Cistoscopia realizada ambulatorialmente demonstrando tumor vesical.

Reinternou no dia23 deabril de2001 para tratamento cirúrgico do tumor vesical nesta mesma data. Realizou tomografia computadorizada abdomino-pélvica préoperatória que evidenciou apenas alteração da bexiga, com paredes irregularmente espessadas, com massa sólida, homogênea, de base larga na parede vesical esquerda com provável infiltração do meato ureteral esquerdo, pouco captante do meio de contraste (figura 3).
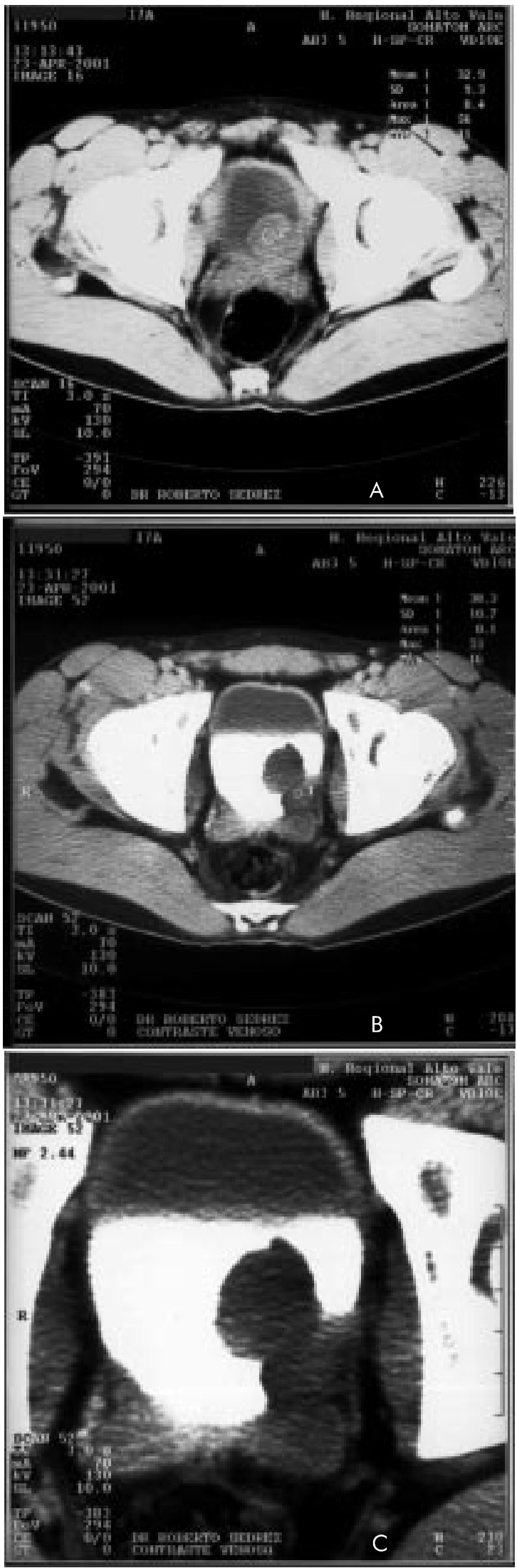

Figura 3: A - Tomografia Computadorizada mostrando massa sólida e homogênea em parede vesical esquerda. B - Fase contrastada demonstrando o tumor vesical de base larga e pouco captante do meio de contraste. C - Imagem ampliada da fase contrastada. 
Iniciou o procedimento com ressecção transuretral, mas devido a reflexo do obturador interno e comprometimento do meato ureteral esquerdo houve conversão para cirurgia a céu aberto. Foi realizada então a ressecção do tumor e cateterização do ureter esquerdo com cateter duplo J. N ão foi realizada linfadenectomia locorregional. Permaneceu internado até a data de 29 de abril de 2001 e neste período manteve-se com sinais vitais estáveis, com provas laboratoriais sem alterações, com melhora da hematúria e clareamento da urina no $5^{\circ}$ dia pós-operatório.

0 resultado do exame anátomo-patológico da peça revelou quadro histológico compatível com paraganglioma extra medular com necessidade de estudo imunohistoquímico (figura 4).

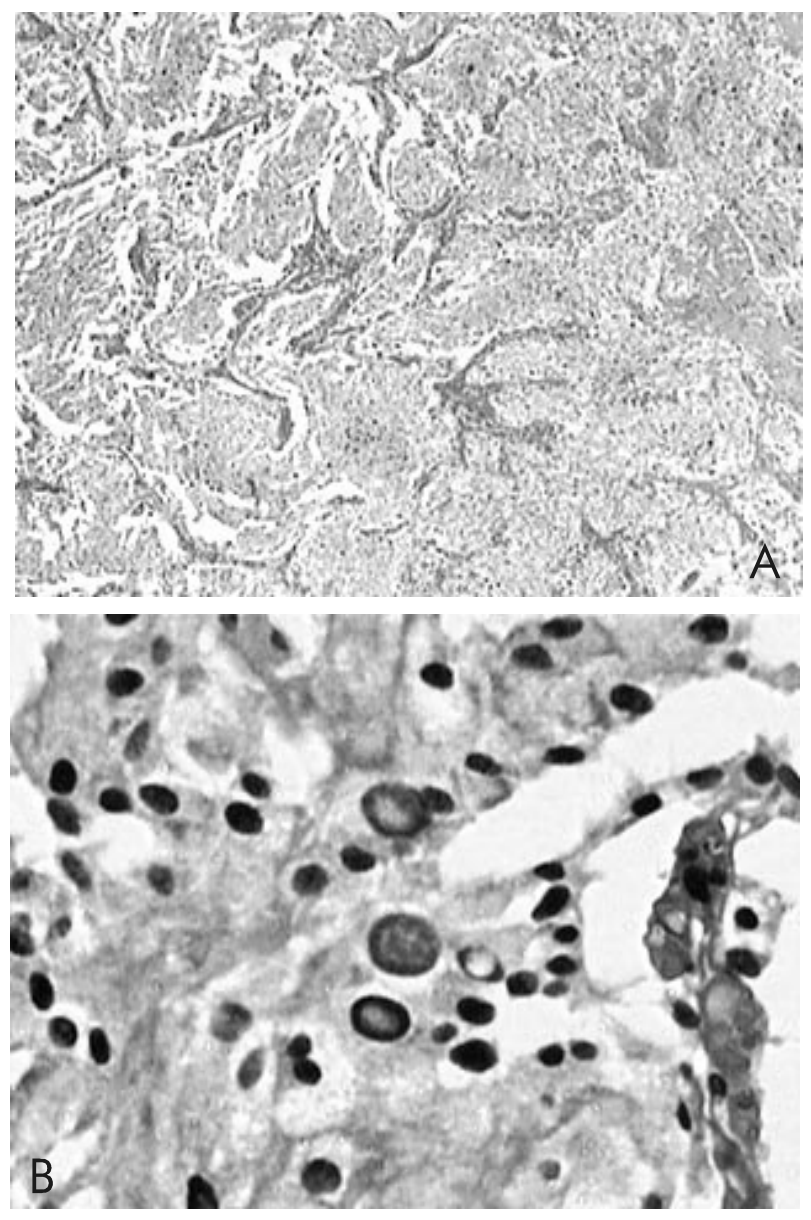

Figura 4: A - Paraganglioma de bexiga com arranjo alveolar (área do tipo Zellballen). B - Paraganglioma de bexiga com presença de pseudo-inclusões nucleares.

0 paciente apresentou boa evolução no pósoperatório sendo retirado o cateter duplo J no dia 24 de maio de 2001.

0 estudo imunohistoquímico foi realizado em 29 de maio de 2001, tendo concluído se tratar de paraganglioma (figura 5).

Em 25 de fevereiro de 2002 realizou cintilografia com iodo-131 metaiodobenzilguanidina (MIGB), que concluiu baixa probabilidade de tumoração da linhagem neuroectodérmica.

Em 01 de abril de 2002, em consulta ambulatorial, o paciente apresentava-se hígido e assintomático.
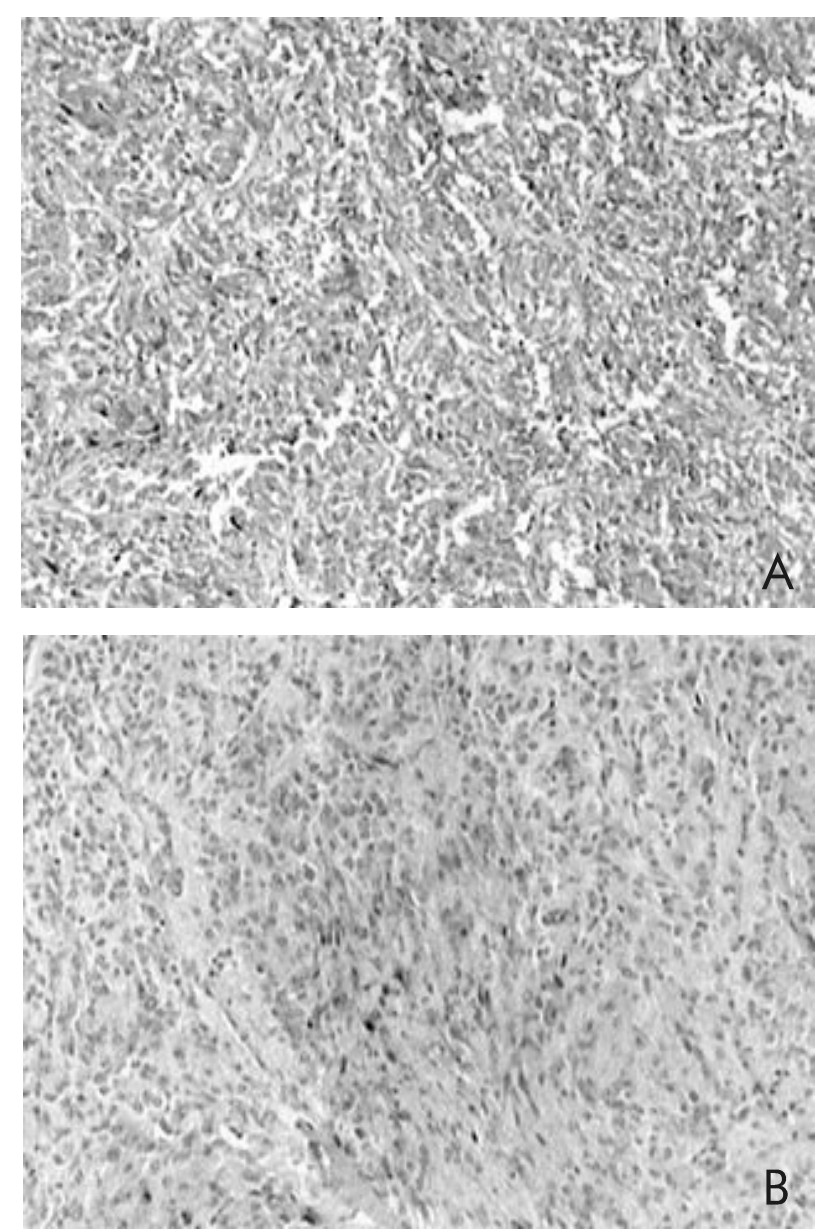

Figura 5: A - Paraganglioma em bexiga urinária (cromogranina). B - Paraganglioma de bexiga (synaptofisina).

\section{DISCUSSÃO}

$\mathrm{N}$ oventa por cento dos feocromocitomas surgem na medula adrenal e os tumores extra adrenais ocorrem em $10 \%$ a $20 \%$ de todos os casos. Esses paragangliomas são multifocais em aproximadamente $10 \%$ dos casos, e parecem surgir de pequenos ninhos de tecido paragangliônico que persistem ao longo do eixo aórtico ${ }^{1}$. 0 itenta por cento dos tumores extra-adrenais se localizam no abdome, na região paraaórtica superior 
(entre o diafragma e as artérias renais inferiores) ${ }^{3}$. A maioria é benigna e o diagnóstico de tumor maligno é estabelecido quando a metástase é encontrada em área onde não se presume ter tecido cromafim, como linfonodos, ossos, fígado e pulmão. A idade de apresentação dos paragangliomas oscila entre 11 e 70 anos, sendo mais freqüente entre a $2^{\text {a }}$ e a $4^{\text {a }}$ décadas de vida, sem predominância de sex $0^{4}$. Sua história natural é variável, com a maioria, especialmente aqueles associados com fibras de pares cranianos (tumores do glomo carotídeo, jugular, timpânico, vagal e aórtico), sendo indolentes por 10 anos $5^{5}$. Em torno de 10\% dos paragangliomas metastatizam para gânglios linfáticos regionais ou à distância, e cerca de $5 \%$ podem ser agressivos localmente 4 . 0 potencial metastático é mais alto para tumores de tronco (mediastinal e retroperitoneal), e os locaismais comuns demetástases são linfonodos, ossose pulmões ${ }^{5}$. Vinteacinquenta por cento dos paragangliomas retroperitoneais têm comportamento maligno 4 . Clinicamente os paragangliomas são classificados em funcionantes e não-funcionantes. O s paragangliomas de bexiga funcionantes (83\%) são localizados normalmente na cúpula e no trígono, em $34,4 \%$ a $41 \%$. D ez a quinze por cento dos paragangliomas de bexiga são malignos ${ }^{1}$. A síndrome miccional, que se caracteriza pela ocorrência de cefaléia, palpitação, hipertensão, hematúria e visão borrada, está presente em $47 \%$ a $77 \%$ dos casos. Esses sintomas aparecem durante ou poucos minutos depois da micção e, geralmente, duram al guns minutos, podendo entretanto persistir por horas. A pressão sangüínea pode ser normal, constantemente elevada ou paroxisticamente elevada. A hematúria está presente em $55 \%$ a $60 \%$ e hipotensão intermitente em $65 \%$ a $70 \%$ dos pacientes ${ }^{1,4}$.

A investigação laboratorial e as técnicas de imagem são necessárias para o diagnóstico do paraganglioma. 0 teste bioquímico de rastreamento mais sensível é a dosagem na urina de catecolaminas, ácido vanilmandélico e metanefrinas. A maior parte dos paragangliomas funcionantes secretam noradrenalina exclusivamente ou como catecolamina predominante. Q uando predomina a epinefrina, deve-se suspeitar de localização intra-adrenal (feocromocitoma) ${ }^{1,4}$.

O s estudos de localização são de custo elevado e nem sempre específicos, mas se constituem em uma técnica diagnóstica relevante. Estes exames são: ultrassonografia (USG), urografia excretora, cistoscopia, tomografia computadorizada (TC), ressonância nuclear magnética (RNM) e a cintilografia com iodo 131$M$ etaiodobenzilguanidina ${ }^{3,4}$.

A cistocopia em mais de $80 \%$ dos casos revela um tumor em submucosa, no trígono ou na cúpula da bexiga, ao contrário da maioria dos tumores de células transicionais da bexiga que envolvem a mucosa ${ }^{1,4}$.

A urografia excretora pode mostrar fal ha no enchimento da bexiga ou obstrução ureteral ${ }^{1,4}$.

A USG e a TC são exames não invasivos inespecíficos, com a vantagem de melhor visualização de lesões menores do que $1 \mathrm{~cm}$ pela TC. A TC é útil para a detecção de tumores supra-renais e a RN M para tumores justavesicais. A RN M pode ser superior à TC, mas a experiência com esse método é limitada ${ }^{1,3,4}$.

A cintilografia através do radiofármaco metaiodobenzilguanidina (131-I M IBG) tem sido aplicada como um método de identificação de tumores originários de tecidos cromafis, tanto adrenais como extra-adrenais, sendo prontamente absorvido por estes tecidos. 0 iodo 131-MIBG tem estrutura molecular semelhante à noradrenalina e sua incorporação no tecido cromafim é feita por mecanismo similar àquele observado com um neurotransmissor ou hormônio. A utilização deste radiofármaco é valiosa para o diagnóstico de localização torácica, sítio este pouco identificado pela tomografia computadorizada, e também no acompanhamento de alguns paragangliomas malignos. 0 método de captação com 131-I M IBG é considerado o mais específico (95\%) para o diagnóstico dos paragangliomas funcionantes podendo detectar tumores múltiplos ou pequenos, sendo o exame mais sensível (85\%) para se detectar doença metastática ou recorrente. 0 papel da cintilografia com iodo-131 MIBG nos tumores não funcionantes é desconhecido $0^{1,3,4}$.

0 tratamento do paraganglioma de bexiga é primariamente cirúrgico, ou seja, ressecção transuretral, cistectomia total ou parcial. Para excluir a hipótese de doença metastática ou multifocal. Recomenda-se a exploração dos linfonodos pélvicos e retroperitoneais e, se a linfadenopatia metastática é confirmada, a cistectomia radical mais linfadenectomia pélvica superior devem ser consideradas. A preparação préoperatória dos pacientes é feita com alfa-bloqueadores e, se necessário, beta-bloqueadores mais hidratação rigorosa para evitar hipotensão após a remoção do tumor. D eve-se monitorar a hipoglicemia e a hipotensão após a retirada do tumor, devido a perda da estimulação adrenérgica no fígado, pâncreas e circulação periférica. A radioterapia e a quimioterapia na doença metastática sintomática devem ser consideradas, porém apresentam resultados pobres. Finalmente, nos últimos anos tem sido utilizado o iodo-131 MIBG como método terapêutico de paliação para o tratamento dos tumores 
derivados da crista neural, podendo aliviar os sintomas, produzir regressões tumorais, mas raramente ser curativo ${ }^{1}$.

0 prognóstico do paraganglioma vesical ainda não é bem conhecido devido ao pequeno número de casos e 0 respectivo seguimento ${ }^{1}$. Análises recentes mostraram que a ploidia do D NA pode ter valor prognóstico. As células sustentaculares, que são vistas freqüentemente nos mais benignos, são vistas raramente ou não são vistas nos mais agressivos ${ }^{2}$. Tentativas de prever 0 comportamento biológico avaliando marcadoresteciduais de atividade celular proliferativa (ex. ordenadores nucleolares argirofílicos, antígeno nuclear de células em proliferação) tem sido inconsistentes. Pela tendência de recidiva, o paciente deve ser acompanhado por longos períodos, devendo ter-se muita cautela quando se fala de cura definitiva 5 .

\section{AGRADECIMENTOS:}

Professor Ilson Blogoslawski - Professor da D isciplina de M etodologia Científica da U niversidade para o Desenvolvimento do Alto Vale do Itajá - U nidavi.

\section{REFERÊNCIAS}

1. Naime FF, Begliomini H, Araújo M C, dos Santos M C, M oraesRSP, D uarte J, et al. Paraglioma debexiga: relato de um caso e revisão da literatura. Rev Bras Cancerol. 1997;43(2):127-32.

2. Cheng L, Leibovich $B C$, ChevilleJC, Ramnani DM , Sebo TJ, N eumann RM , et al. Paraganglioma of theurinary bladder: can biological potential be predicted? Cancer. 2000;88(4):844-52.

3. ChesaPonceN, ArmasM olinaJ, PonceSocorro J, D el Rosario M edina J, Artiles H ernandez J L, M artin Betancor D, et al. Paraganglioma vesical. Presentación de um caso y revisión de la literaturaespañola. ActasU rol Esp. 1997;21(1):52-6.

4. Tato Rodriguez J, Blanco Parra M, N ovas Castro S, Cimadevila C ovelo A, Cameselle-Teijeiro J. Paraganglioma vesical asintomático. A portación de um caso. Actas U rol Esp. 1995;19(5):408-13.

5. Pastel SR, W inchester DJ, Benjamin RS. A 15-year experience with chemotherapy of patients with paraganglioma. Cancer. 1995;76(8):1476-80.

6. Rhaman SI, Matthews LK, Shaikh H, Townell N H . Primary paraganglioma of the bladder in a 14-year-old boy. $\mathrm{Br}$ J U rol. 1995;75(5):682-3. 